\title{
Scoping review protocol on nonpharmacological interventions for possible sarcopenia or sarcopenia in community-dwelling older adults
}

Ya Shi ( $\nabla$ ya.shi@postgrad.manchester.ac.uk)

The University of Manchester https://orcid.org/0000-0002-1506-9022

\section{Emma Stanmore}

The University of Manchester

Chris Todd

The University of Manchester

\section{Research Article}

Keywords: Sarcopenia, Geriatrics, Older adults, Nonpharmacological intervention, Community, Scoping review

Posted Date: January 20th, 2022

DOI: https://doi.org/10.21203/rs.3.rs-1246504/v1

License: (c) (i) This work is licensed under a Creative Commons Attribution 4.0 International License. Read Full License 


\title{
Scoping review protocol on nonpharmacological interventions for possible sarcopenia or sarcopenia in community-dwelling older adults
}

\begin{abstract}
Background: Preventing sarcopenia earlier in community has become a new recommendation in recent years. Several nonpharmacological interventions to reduce the risk of sarcopenia in community-dwelling older people have been proposed, therefore there is a need to identify which of these interventions are effective. This scoping review will summarise the nature and extent of the existing literature that describes and examines nonpharmacological interventions for older adults with possible sarcopenia or sarcopenia in community.
\end{abstract}

Methods: Seven stages of methodology framework which originally proposed by Arksey and O'Malley and then enhanced by Levac et al and Daudt et al will be used. A search will be conducted in the following databases: Embase, Medline on Ovid, PsycINFO, CINAHL, All EMB Reviews on Ovid, Web of Science, Scopus, CBM, CNKI, WANFANG, VIP. Grey literature will also be searched from Google scholar. Searching will be restricted in date from January 2010 to December 2021 and in English and Chinese language only. Screening will be focused on published research articles and prospectively registered trials, including all the quantitative and qualitative study design. PRISMAScR will be followed for delineating the search decision process. The findings will be synthesised both quantitatively and qualitatively and classified using key conceptual categories. Moreover, it will be assessed whether the studies identified have been included in systematic reviews or metaanalyses, and the research gaps and opportunities will also be identified and summarised.

Discussion: The planned scoping review will enable to identify research status and gaps existing in literatures, which would be beneficial for detecting currently insufficient areas and developing new evidence-based practices of non-pharmacological interventions to prevent sarcopenia among older adults. To our knowledge, this protocol is the first on this topic.

Systematic review registration: Open Science Framework (OSF) Registration DOI 10.17605/OSF. IO/8BMNR

Key words: Sarcopenia; Geriatrics; Older adults; Nonpharmacological intervention; Community; Scoping review 


\section{Background}

\subsection{Rationale}

According to new revised European consensus, sarcopenia is a muscle disease or muscle failure rooted in adverse muscle changes that accrue across a lifetime, which can be divided into three levels: possible; confirmed or severe sarcopenia[1]. Possible sarcopenia is identified by using low muscle strength as the primary parameter and is suggested to be an important boundary to trigger assessment of causes and start intervention in medical practice. In addition, confirmed sarcopenia is diagnosed by low muscle strength and low muscle quantity/quality, whereas if low physical performance is also confirmed, it should be considered as severe sarcopenia[1, 2].

Sarcopenia is a relatively common muscle disease with a very high prevalence rate, which varies widely according to country, region, age, gender, comorbid disease, etc. A recent systematic review and meta-analysis demonstrates that the global prevalence of sarcopenia varies between $10 \%$ and $27 \%$, with the highest and lowest prevalence were observed in Oceania and Europe respectively [3]. A study using a predictive model estimated the number of sarcopenia patients would dramatically rise in Europe from 10,869,527 19,740,527 in 2016 to 18,735,173 32,338,990 in 2045 (72.4\% and 63.8\% increase)[4]. In addition, with regional and age-related variations, another review reported the prevalence of sarcopenia to be $1 \sim 29 \%$ in community-dwelling populations, $14 \sim 33 \%$ in long-term care populations and $10 \%$ in the only acute hospital-care population examined[5]. Moreover, Pagotto and Silveira[6] found the prevalence were different in genders by using dual-X-ray absorptiometry, ranging from $2.2 \%$ to $95 \%$ in males and from $0.1 \%$ to $33.9 \%$ in females. As a comorbid disease, the prevalence of sarcopenia in individuals with cardiovascular disease, dementia, diabetes mellitus, respiratory diseases were $31.4 \%, 26.4 \%, 31.1 \%, 26.8 \%$ respectively[7].

Sarcopenia can be affected by a variety of risk factors. Older age may be the most important among numerous reported risk factors[1]. However, household status, lifestyle, physical inactivity, poor nutritional and dental status, and diseases (such as osteoporosis, cardiac disease, respiratory disease) were also independently associated with sarcopenia [2, 8, 9]. In particular, the likelihood of developing sarcopenia is closely related to several cardiometabolic risk factors, including diabetes, hypertension, and dyslipidemia[2].

Optimal care for people with sarcopenia is essential because it may result in high personal, social and economic burdens if untreated [10]. For the individual, sarcopenia is associated with increased risk of falls and fractures [11, 12]; impairs the ability to perform daily activities [13]; leads to mobility disorders[14]; and contributes to lowered quality of life[15], loss of independence or need for long-term care placement [16-18], and even death[19]. In financial terms, sarcopenia is costly to healthcare systems. The presence of sarcopenia is also related to increased risk of hospitalisation and increased cost of care during hospitalisation[20]. Among older adults who are hospitalised, those with sarcopenia on admission tend to have higher hospital costs (more than five times) than those without sarcopenia[21]. Results of a large-scale, community-based study in the Czech Republic showed that direct healthcare costs were more than twice as high for older people with sarcopenia than for those without[22]. All of these adverse outcomes lead to high utility levels of health and social resources and adversely affect the healthy ageing of society.

Hence, more and more attention has been paid to the prevention and treatment of sarcopenia in recent years around the world. In early 2018, the European Working Group on Sarcopenia in Older People (EWGSOP2) updated the original definition in order to reflect scientific and clinical 
evidence that has built over the last decade. EWGSOP2's updated recommendations aim to increase awareness of sarcopenia and its risk. With these new recommendations, EWGSOP2 calls for healthcare professionals who treat patients at risk for sarcopenia to take actions that will promote early detection and treatment[1]. Besides, to promote healthy ageing, both EWGSOP2 and the Asian Working Group for Sarcopenia (AWGS) (2019) have defined a new notion of possible sarcopenia and recommend it to primary health care and preventive service users, which will contribute to a higher awareness of sarcopenia prevention and interventions in diverse health care settings $[1,2]$.

There are a number of studies on the development of pharmacological and nonpharmacological strategies against sarcopenia but there is considerable heterogeneity of both definitions and interventions. Concerning pharmacological therapeutics, studies on sarcopenia have more focused on treatments such as myostatin/ActR2 signaling inhibitors[23, 24], exercise mimetics[25], anabolic hormones[26], specific nutrients[27], phytochemicals[27, 28]. Drug names include Bimagrumab (antibody), Sarconeos (natural active ingredients), ARM-210 (small molecule), NT-1654 (fragment of neural agrin), AVGN7 (gene therapy), VB-102 (protein)[29] and more. However, to date, there remain no US Food and Drug Administration (FDA) approved drugs for the treatment of sarcopenia[29]. It should be emphasised that in previous literature, nutritional regulation was one of the most common interventions for sarcopenia, which actually contained both pharmacological and nonpharmacological elements. According to the food synergy concept which is helpful to distinguish between a food and a drug, many supplements that derived from food are isolated substances and could be classified as drugs, and foods enriched with an isolated substance can be seen as drugs delivered via a food[27]. Therefore, to make this scoping review more rigorous, dietary supplements including specific nutrients (e.g. vitamin, minerals, amino acid, aliphatic acid) and/or phytochemicals (e.g. carotenoids, ursolic acid, tomatidine) will be classified as pharmaceutical. If supplements are for "the prevention, cure, mitigation, and therapeutic treatment of disease", then they are considered to be drugs and as such, are subject to regulation and approval by the FDA.

Regarding nonpharmacological interventions for sarcopenia, types of these intervention strategies can be divided into several main categories: physical activity, dietary modification (overall food intake and dietary patterns), health education and emotional support. Among them, the first two approaches in independent or joint intervention are usual. The modalities of physical activity described in the literature are numerous [30-34] and different types of exercise can stimulate variable, but specific, responses in muscle functions[35]. However, there were discrepancies in the selection and combination of exercise modes, exercise intensity, total repetitions, rest periods, training dose, regularity and progression among different studies. As for dietary modification, overall food intake[30, 36-39] and dietary patterns change[40-42] have been concerned in muscle health and sarcopenia prevention in recent years, but there is lack of holistic understanding about the types, characteristics and intervention effectiveness among all these different foods or dietary patterns until now.

Compared with physical activity and nutritional regulation, the studies that mentioned health education and emotional support were rare. In terms of health education, only a few studies mentioned health education methods and tested the intervention effects, which were significantly lower than that of physical activity and/or nutrition groups[43-45]. However, it might be surprising that the health education components directly related to the sarcopenia such as causes, risk factors, preventive measures, treatment methods were missing in these studies[32, 44, 46-48]. Instead, they 
tend to focus specifically on dementia prevention, prevention of bank transfer fraud, cognitive function, long-term care insurance, music therapy, oral care, etc. As a result, in the absence or the ineffective ways of health education, the participants may not have a deep understanding of sarcopenia neither its harm, which may be the main reason that the effects of health education in these studies were not satisfactory. In addition, studies also shown that sarcopenia were associated with depressive mood[49-52] and bipolar disorder[53], which calls for emotional support in these areas.

Although there already exists several reviews[18, 54, 55], they only focused on physical activity and/or nutritional regulation without age or health care settings restriction, and did not include health education and emotional support or target possible sarcopenia. Besides, due to nutritional regulation contains pharmacological and nonpharmacological interventions, there is still no evidence in sarcopenia literature to distinguish them explicitly. In addition, as a new classification of sarcopenia is recommended by the EWGSOP2 and the (AWGS) 2019, whether there are differences among nonpharmacological interventions for possible, confirmed and severe sarcopenia is still a blank area in current researches. Therefore, it is essential to carry out a scoping review on nonpharmacological interventions for possible sarcopenia or sarcopenia in community-dwelling older adults at present, which will be a solid foundation and evidence for the development and perfection of nonpharmacological interventions for preventing and treating sarcopenia.

\subsection{Objectives}

The purpose of this scoping review is to identify and explore the evidence describing and examining nonpharmacological interventions for older adults with possible sarcopenia or sarcopenia in community setting. In addition, we will explore if there is any difference among nonpharmacological interventions for possible, confirmed and severe sarcopenia. Furthermore, we will investigate whether each intervention found has been included in any systematic reviews or meta-analyses to decide whether it is necessary to conduct a systematic review or an overview of systematic reviews on this topic in the next research stage. The final goals of this review are to direct future research efforts by identifying gaps and limitations in the existing literatures in the relevant field.

\section{Methods/design}

This protocol was developed using the seven stages of scoping review methodology framework which was originally proposed by Arksey and O'Malley[56] and then enhanced by Levac et al[57] and Daudt et al[58], including: (1) identification of research questions, (2) identification of relevant studies, (3) selection of relevant studies, (4) Extracting and charting data, (5) Collating, summarising and reporting results, (6) Consultation, and (7) Transferring knowledge.

\subsection{Identifying research questions}

This scoping review will answer the following questions:

- What study types exist in terms of nonpharmacological interventions for older adults with possible sarcopenia or sarcopenia in community settings?

- What are the types, durations, frequencies and timings of nonpharmacological interventions effective for older adults with possible sarcopenia or sarcopenia in community settings?

- What are the differences among nonpharmacological interventions for possible, confirmed and severe sarcopenia? 
- What are the challenges and barriers to preventing sarcopenia using nonpharmacological interventions within older adults in community setting?

- Which studies have been included in a systematic review/meta-analysis, and based on this, is it necessary to conduct a systematic review or an overview of systematic reviews in the next research stage?

\subsection{Identifying relevant studies}

The team collaborated to plan a search strategy in consultation with a professional librarian to identify a comprehensive list of relevant literature specific to nonpharmacological interventions for older adults with possible sarcopenia or sarcopenia in community settings. The electronic search for literature will focus on retrieving published articles in peer-reviewed scientific journals and prospectively registered trials in registration website by a systematic search of the following databases: Embase, Medline on Ovid, Psychological Information (PsycINFO), Cumulative Index to Nursing and Allied Health Literature (CINAHL), All Evidence Based Medicine Reviews (All EMB Reviews) on Ovid, Web of Science, Scopus, Chinese Biomedical Literature Database (CBM), Chinese National Knowledge Infrastructure (CNKI), Wan Fang Database (WANFANG), Chinese Science and Technology Periodical Database (VIP). Grey literature will also be searched from Google scholar. Searching will be restricted in date from January 2010 to December 2021 and in English and Chinese language only.

Two researchers will independently perform the literature search and eligibility assessments. A pilot search will be performed on MEDLINE to identify relevant keywords contained in the title, abstract and subject descriptors. We will use the following search terms related to nonpharmacological interventions and older adults with possible sarcopenia or sarcopenia in the community, with various combinations in each electronic database while using controlled vocabulary with the Boolean operators AND and OR. We will use appropriate subject headings (eg, Medical Subject Headings) whenever possible. A copy of the search strategies and the preliminary searches results in each electronic database will be saved.

The basic search terms used will be:

1. (Sarcopeni*)

2. (Older adult* OR Older person OR Elder* OR Geriatric* OR Senior* OR Veteran* OR Aged OR Aged, 80 over OR Oldest old OR Age, Eld* OR Nonagenarian* OR Octogenarian* OR Centenarian*)

\section{1 AND 2}

\subsection{Selecting relevant studies}

The selection process of relevant studies will follow two stages of screening. First step, the selected documents will be integrated into Endnote software to eliminate duplicates and then be transferred to another software, Rayyan, to conduct initial review. The initial screening of titles, abstracts and keywords will be done independently by two reviewers (YS and YMT) to assess relevance of each study, and two reviewers will discuss the results once screening is completed. Resolving disagreements will be attempted first by two reviewers, if necessary, a third member (ES) of the research team will be consulted to reach consensus. The second step will go through a fulltext review. Two reviewers (YS and YMT) will independently assess the articles to determine whether they meet the inclusion criteria. Disagreements regarding inclusion will be discussed and 
resolved by consensus with a third member (CT) of the research team adjudicating articles.

Table 1 describes the inclusion and exclusion criteria of study selection, following three categories of the Population, Concept and Context[59]. Inclusion criteria are studies that focus on: (1) older individuals 60 years of age or older (or where the mean or median age of the study sample was 60 years of age or older) who had possible sarcopenia or sarcopenia diagnosis. Diagnostic criteria refer to the requirements of the EWGSOP2[1] and the AWGS2019[2]; (2a) describing or reporting the types, durations, frequencies or timings of nonpharmacological interventions for preventing sarcopenia; (2b) evaluating the effectiveness of different nonpharmacological interventions for preventing sarcopenia, and the characteristics and contexts contributing to positive outcomes or experiences; (2c) reporting the challenges and barriers of preventing sarcopenia using nonpharmacological interventions within older adults; (3) studies conducted in community settings. Research articles using quantitative or qualitative study design or reviews will be included to support the greater breadth of this scoping review.

A Preferred Reporting Items for Systematic Reviews and Meta-Analyses flow diagram for the scoping reviews (PRISMA-ScR) will be used to delineate the search decision process[60], which will include the searching results, removal of duplicate citations, study selection, full retrieval and additions from reference list searching and final selection for inclusion.

\subsection{Extracting and charting data}

A standardised form developed by our research team will be used to extract data from the articles that meet the inclusion criteria. All relevant data will be included to answer the scoping review questions that we originally set. The basic content to be charted will include: description of study characteristics (eg. authors, type of study design, publication year, country, the geographical location in which the research was conducted, aims/purpose, methodology and sample size), description of study populations (eg. age, gender, residence, ethnicity, cultural background, cognition and comorbidity), description of nonpharmacological interventions and control conditions (eg. type of intervention, duration, frequency, timing), effectiveness (eg. process, impact and outcome), the challenges and barriers. The form will be piloted by two reviewers (YS and YMT) on three studies before formal use, and differences in charting will be resolved by a third member (CT/ES) of the research team.

Two reviewers (YS and YMT) will extract the data independently and any discrepancies will be resolved through discussion and consensus with the third member (CT/ES). Any data found to be missing in the report of the studies will be requested directly from the original authors. According to the scoping review's methodology, the aim is to identify and describe the evidence; therefore, the quality of individual studies will not be assessed.

\subsection{Collating, summarising and reporting results}

First, the extracted data will be summarised to provide a description by using descriptive statistics, which will be reported in tables and/or in narrative forms. Second, in accordance with recommendations from the literature[61], a method of parallel-results convergent synthesis design will be used to synthesise quantitative and qualitative data, where both types of evidence will be analysed and presented separately, with integration occurring during the interpretation of results. The strength of this method is to provide a synthesis strategy for addressing multiple complementary review questions pertaining to our broadly covered topic with regards to nonpharmacological 
interventions for older adults with possible sarcopenia or sarcopenia in community setting, thereby providing a big map of existing evidence and research gaps.

For quantitative data, frequency distribution analysis will be used to describe the data and thematic synthesis will also be used to contextualise the findings where appropriate. For qualitative data, narrative synthesis and thematic synthesis of the findings will both used to be conducted for review different research questions. As for thematic synthesis, major themes will be identified and developed across included studies. Finally, research gaps and opportunities will also be identified and summarised. The review results may be presented as a 'numerical summary', 'narrative summary', 'table', 'conceptual map' and/or 'schematic representation' of the data. Additional presentation formats will be decided after data extraction, so as to make sure the results are clear and visually compelling to readers.

\subsection{Consultation}

A consultation team formed of different stakeholders will be put in place, which will comprise older adults with possible sarcopenia or sarcopenia, community health caregivers, occupational physicians and researchers in the field of sarcopenia. The consultation will take 'focus group' form and each participant will bring unique expertise that will enrich the analysis perspectives of relevant topic. The consulting purpose is to verify applicability of the results and validity of the contents of the scoping review, so as to provide important information and essential foundation for planning research proposal for next stage.

\subsection{Transferring knowledge}

New knowledge related to nonpharmacological interventions for older adults with possible sarcopenia or sarcopenia in community settings will be generated from this study, which is then important and useful to knowledge users, including patients, caregivers, medical professionals and researchers. On the one hand, an internet group containing possible sarcopenia or sarcopenia patients and their caregivers will be developed and the meaningful results explained in plain language will be shared with them through email or website. On the other hand, another internet group containing medical professionals and researchers working closely with sarcopenia will be constructed and recommended the professional study results, so as to improve their clinical practice with the best evidence. All these modalities of knowledge transfer will be submitted to the consultation team for validation.

\section{Discussion}

The scoping review proposed in this protocol will synthesise knowledge to foster development of practices to non-pharmacological interventions for older adults with possible sarcopenia or sarcopenia in community setting. To our knowledge, this scoping review protocol is the first on this topic. The analysis and synthesis of quantitative and qualitative studies through the seven-step systematic scoping review method will enable to identify research status and gaps existing in literatures, which would be beneficial for detecting currently insufficient areas and developing new evidence-based practices of non-pharmacological interventions to prevent sarcopenia among older adults. Finally, the proposed, including stakeholders' consultation and knowledge transfer activities, will be tested in this study, contributing to the advancement of knowledge in the methodological field of literature reviews. 


\section{Strengths and limitations of this study}

- This scoping review will contribute to identifying non-pharmacological interventions for possible sarcopenia or sarcopenia in community-dwelling older adults.

- This is a review-based approach to mapping quantitative and qualitative literatures on a complex and broad topic.

- The inclusion of grey literature helps to reduce publication bias.

- No restrictions will be applied in terms of the date of publication.

- As a scoping review, the quality of individual studies will not be assessed.

- This review focuses on studies written in the English and Chinese languages and could miss out on relevant literature published in other languages.

\section{References}

[1] Cruz-Jentoft AJ, Bahat G, Bauer J et al. Sarcopenia: revised European consensus on definition and diagnosis. Age Ageing 2019; 48(1):16-31.

[2] Chen LK, Woo J, Assantachai P et al. Asian Working Group for Sarcopenia: 2019 Consensus Update on Sarcopenia Diagnosis and Treatment. J Am Med Dir Assoc 2020; 21(3):300-307.

[3] Petermann-Rocha F, Balntzi V, Gray SR et al. Global prevalence of sarcopenia and severe sarcopenia: a systematic review and meta-analysis. Journal of cachexia, sarcopenia and muscle 2021. [4] Ethgen O, Beaudart C, Buckinx F et al. The Future Prevalence of Sarcopenia in Europe: A Claim for Public Health Action. Calcif Tissue Int 2017; 100(3):229-234.

[5] Cruz-Jentoft AJ, Landi F, Schneider SM et al. Prevalence of and interventions for sarcopenia in ageing adults: a systematic review. Report of the International Sarcopenia Initiative (EWGSOP and IWGS). Age Ageing 2014; 43(6):748-759.

[6] Pagotto V, Silveira EA. Methods, diagnostic criteria, cutoff points, and prevalence of sarcopenia among older people. Scientific World Journal 2014; 2014:231312.

[7] Pacifico J, Geerlings MAJ, Reijnierse EM et al. Prevalence of sarcopenia as a comorbid disease: A systematic review and meta-analysis. Exp. Gerontol. 2020; 131:19.

[8] Wong A, Zhu D, Kraus D, Tham T. Radiologically Defined Sarcopenia Affects Survival in Head and Neck Cancer: A Meta-Analysis. Laryngoscope 2021; 131:333-341.

[9] Bone AE, Hepgul N, Kon S, Maddocks M. Sarcopenia and frailty in chronic respiratory disease. Chron Respir Dis. 2017; 14(1):85-99.

[10] Mijnarends DM, Luiking YC, Halfens RJG et al. Muscle, Health and Costs: A Glance at their Relationship. J Nutr Health Aging. 2018; 22(7):766-773.

[11] Bischoff-Ferrari HA, Orav JE, Kanis JA et al. Comparative performance of current definitions of sarcopenia against the prospective incidence of falls among community-dwelling seniors age 65 and older. Osteoporosis Int. 2015; 26(12):2793-2802.

[12] Schaap LA, van Schoor NM, Lips P, Visser M. Associations of Sarcopenia Definitions, and Their Components, With the Incidence of Recurrent Falling and Fractures: The Longitudinal Aging Study Amsterdam. The journals of gerontology. Series A, Biological sciences and medical sciences 2018; 73(9):1199-1204.

[13] Malmstrom TK, Miller DK, Simonsick EM et al. SARC-F: a symptom score to predict persons with sarcopenia at risk for poor functional outcomes. J Cachexia Sarcopenia Muscle. 2016; 7(1):2836.

[14] Morley JE, Abbatecola AM, Argiles JM et al. Sarcopenia with limited mobility: an international 
consensus. J Am Med Dir Assoc 2011; 12(6):403-409.

[15] Beaudart C, Biver E, Reginster JY et al. Validation of the SarQoL(R), a specific health-related quality of life questionnaire for Sarcopenia. Journal of cachexia, sarcopenia and muscle 2017; 8(2):238-244.

[16] Dos Santos L, Cyrino ES, Antunes M et al. Sarcopenia and physical independence in older adults: the independent and synergic role of muscle mass and muscle function. Journal of cachexia, sarcopenia and muscle 2017; 8(2):245-250.

[17] Akune T, Muraki S, Oka H et al. Association of physical activities of daily living with the incidence of certified need of care in the long-term care insurance system of Japan: the ROAD study. Journal of Orthopaedic Science 2014; 19(3):489-496.

[18] Michal S, Bohannon RW, Lenka S et al. Relationship between sarcopenia and physical activity in older people: a systematic review and meta-analysis. Clin Interv Aging 2017; 12(5):835-845.

[19] De Buyser SL, Petrovic M, Taes YE et al. Validation of the FNIH sarcopenia criteria and SOF frailty index as predictors of long-term mortality in ambulatory older men. Age Ageing 2016; 45(5):602-608.

[20] Cawthon PM, Lui LY, Taylor BC et al. Clinical Definitions of Sarcopenia and Risk of Hospitalization in Community-Dwelling Older Men: The Osteoporotic Fractures in Men Study. The journals of gerontology. Series A, Biological sciences and medical sciences 2017; 72(10):1383-1389. [21] Antunes AC, Araujo DA, Verissimo MT, Amaral TF. Sarcopenia and hospitalisation costs in older adults: a cross-sectional study. Nutr Diet 2017; 74(1):46-50.

[22] Steffl M, Sima J, Shiells K, Holmerova I. The increase in health care costs associated with muscle weakness in older people without long-term illnesses in the Czech Republic: results from the Survey of Health, Ageing and Retirement in Europe (SHARE). Clin Interv Aging 2017; 12:20032007.

[23] Saitoh M, Ishida J, Ebner N, al. e. Myostatin inhibitors as pharmacological treatment for muscle wasting and muscular dystrophy. JCSM Clinical Reports 2017; 2(1):1-10.

[24] Rooks D, Praestgaard J, Hariry S et al. Treatment of Sarcopenia with Bimagrumab: Results from a Phase II, Randomized, Controlled, Proof-of-Concept Study. Journal of the American Geriatrics Society 2017; 65(9):1988-1995.

[25] Narkar VA, Downes M, Yu RT et al. AMPK and PPAR $\delta$ Agonists Are Exercise Mimetics. Cell 2008; 134:405-415.

[26] Papanicolaou DA, Ather Sn Fau - Zhu H, Zhu H Fau - Zhou Y et al. A phase IIA randomized, placebo-controlled clinical trial to study the efficacy and safety of the selective androgen receptor modulator (SARM), MK-0773 in female participants with sarcopenia. The journal of nutrition, health \& aging 2013; 17:533-543.

[27] Jacobs DJ, Tapsell LC. Food, not nutrients, is the fundamental unit in nutrition. Nutr Rev 2007; 65(10):439-450.

[28] Kunkel SD, Suneja M, Ebert SM et al. mRNA expression signatures of human skeletal muscle atrophy identify a natural compound that increases muscle mass. Cell Metab 2011; 13(6):627-638.

[29] Kwak JY, Kwon KS. Pharmacological Interventions for Treatment of Sarcopenia: Current Status of Drug Development for Sarcopenia. Ann Geriatr Med Res 2019; 23(3):98-104.

[30] Granic A, Hurst C, Dismore L et al. Milk and resistance exercise intervention to improve muscle function in community-dwelling older adults at risk of sarcopenia (MIlkMAN): protocol for a pilot study. BMJ Open. 2019; 9(10):e031048. 
[31] Jung WS, Kim SW, Kim JW, Park HY. Resistance Training in Hypoxia as a New Therapeutic Modality for Sarcopenia-A Narrative Review. Life (Basel) 2021; 11(2):106.

[32] Kim H, Kim M, Kojima $\mathrm{N}$ et al. Exercise and Nutritional Supplementation on CommunityDwelling Elderly Japanese Women With Sarcopenic Obesity: A Randomized Controlled Trial. J Am Med Dir Assoc 2016; 17(11):1011-1019.

[33] Gonzalez A, Valero-Breton M, Huerta-Salgado C et al. Impact of exercise training on the sarcopenia criteria in non-alcoholic fatty liver disease: a systematic review and meta-analysis. Eur J Transl Myol 2021; 31(1):9630.

[34] Rondanelli M, Klersy C, Terracol G et al. Whey protein, amino acids, and vitamin D supplementation with physical activity increases fat-free mass and strength, functionality, and quality of life and decreases inflammation in sarcopenic elderly. The American journal of clinical nutrition 2016; 103(3):830-840.

[35] Robinson MM, Dasari S, Konopka AR et al. Enhanced Protein Translation Underlies Improved Metabolic and Physical Adaptations to Different Exercise Training Modes in Young and Old Humans. Cell Metab 2017; 25(3):581-592.

[36] Maltais ML, Ladouceur JP, Dionne IJ. The Effect of Resistance Training and Different Sources of Postexercise Protein Supplementation on Muscle Mass and Physical Capacity in Sarcopenic Elderly Men. Journal of Strength and Conditioning Research 2016; 30(6):1680.

[37] Kim J, Lee Y, Kye S et al. Association between healthy diet and exercise and greater muscle mass in older adults. Journal of the American Geriatrics Society 2015; 63(5):886-892.

[38] Bradlee ML, Mustafa J, Singer MR, Moore LL. High-Protein Foods and Physical Activity Protect Against Age-Related Muscle Loss and Functional Decline. The journals of gerontology. Series A, Biological sciences and medical sciences 2017; 73(1):88-94.

[39] Radavelli-Bagatini S, Zhu K, Lewis JR, Prince RL. Dairy food intake, peripheral bone structure, and muscle mass in elderly ambulatory women. J Bone Miner Res 2014; 29(7):1691-1700.

[40] McClure R, Villani A. Mediterranean Diet attenuates risk of frailty and sarcopenia: New insights and future directions. JCSM Clinical Reports 2017; 2(2):1-17.

[41] Chan R, Leung J, Woo J. A Prospective Cohort Study to Examine the Association Between Dietary Patterns and Sarcopenia in Chinese Community-Dwelling Older People in Hong Kong. J Am Med Dir Assoc 2016; 17(4):336-342.

[42] Kuczmarski MF, Mason MA, Beydoun MA et al. Dietary Patterns and Sarcopenia in an Urban African American and White Population in the United States. Journal of Nutrition in Gerontology and Geriatrics 2013; 13:291-316.

[43] Chan DD, Tsou HH, Chang CB et al. Integrated care for geriatric frailty and sarcopenia: a randomized control trial. J Cachexia Sarcopenia Muscle. 2017; 8(1):78-88.

[44] Landi F, Cesari M, Calvani R et al. The "Sarcopenia and Physical fRailty IN older people: multi-componenT Treatment strategies" (SPRINTT) randomized controlled trial: design and methods. Aging Clin Exp Res. 2017; 29(1):89-100.

[45] de Mello RG, Dalla Corte RR, Gioscia J, Moriguchi EH. Effects of physical exercise programs on sarcopenia management, dynapenia, and physical performance in the elderly: a systematic review of randomised clinical trials. J Aging Res. 2019; 2019(11):1959486.

[46] Kim HK, Suzuki T, Saito K et al. Effects of exercise and amino acid supplementation on body composition and physical function in community-dwelling elderly Japanese sarcopenic women: a randomized controlled trial. Journal of the American Geriatrics Society 2012; 60(1):16-23. 
[47] Osuka Y, Kojima N, Wakaba K et al. Effects of resistance training and/or beta-hydroxy-betamethylbutyrate supplementation on muscle mass, muscle strength and physical performance in older women with reduced muscle mass: protocol for a randomised, double-blind, placebo-controlled trial. BMJ Open 2019; 9(2):e025723.

[48] Liu CK, Leng X, Hsu FM et al. The impact of sarcopenia on a physical activity intervention: the Lifestyle Interventions and Independence for Elders Pilot Study (LIFE-P). The journal of nutrition, health \& aging 2014; 18(1):59-64.

[49] Yuenyongchaiwat K, Boonsinsukh R. Sarcopenia and Its Relationships with Depression, Cognition, and Physical Activity in Thai Community-Dwelling Older Adults. Curr Gerontol Geriatr Res 2020; 2020:8041489.

[50] Chen L, Sheng Y, Qi H et al. Correlation of sarcopenia and depressive mood in older community dwellers: a cross-sectional observational study in China. BMJ Open 2020; 10(9): e038 089.

[51] Nishikawa H, Enomoto H, Yoh K et al. Association between Sarcopenia and Depression in Patients with Chronic Liver Diseases. J Clin Med 2019; 8(5):634.

[52] Lee I, Cho J, Hong $\mathrm{H}$ et al. Sarcopenia Is Associated with Cognitive Impairment and Depression in Elderly Korean Women. Iran J Public Health 2018; 47(3):327-334.

[53] Bulbul F, Koca I, Tamam L et al. The Prevalence of Sarcopenia in Bipolar Disorder. Neuropsychiatr Dis Treat 2020; 16:915-921.

[54] Beaudart C, Dawson A, Shaw SC et al. Nutrition and physical activity in the prevention and treatment of sarcopenia: systematic review. Osteoporosis Int. 2017; 28(6):1817-1833.

[55] Bao W, Sun Y, Zhang T et al. Exercise Programs for Muscle Mass, Muscle Strength and Physical Performance in Older Adults with Sarcopenia: A Systematic Review and Meta-Analysis. Aging Dis 2020; 11(4):863-873.

[56] Arksey H, O'Malley L. Scoping studies: towards a methodological framework. International Journal of Social Research Methodology 2005; 8(1):19-32.

[57] Levac D, Colquhoun H, O'Brien KK. Scoping studies: advancing the methodology. Implementation Science 2010; 5(1):69.

[58] Daudt HM, van Mossel C, Scott SJ. Enhancing the scoping study methodology: a large, interprofessional team's experience with Arksey and O'Malley's framework. BMC Medical Research Methodology 2013; 13:48.

[59] Peters MD, Godfrey CM, Khalil $\mathrm{H}$ et al. Guidance for conducting systematic scoping reviews. Int J Evid Based Healthc 2015; 13(3):141-146.

[60] Tricco AC, Lillie E, Zarin W et al. PRISMA Extension for Scoping Reviews (PRISMA-ScR): Checklist and Explanation. Ann Intern Med 2018; 169(7):467-473.

[61] Hong QN, Pluye P, Bujold M, Wassef M. Convergent and sequential synthesis designs: implications for conducting and reporting systematic reviews of qualitative and quantitative evidence. Syst Rev 2017; 6(1):61. 


\section{Supplementary Files}

This is a list of supplementary files associated with this preprint. Click to download.

- 4.Table.pdf 